\title{
Simple continuous suture to strengthen the closure of intra-muscle used in the removal of uni-portal video-assisted thoracoscopic surgery thoracic drainage tube
}

\author{
Yuanyuan Xu, Zhiyi Guo, Jia Huang, Ruijun Liu, Junwei Ning, Liang Feng, Qiang Tan \\ Shanghai Lung Tumor Clinical Medical Center, Shanghai Chest Hospital, Shanghai Jiao Tong University (SJTU), Shanghai 200030, China \\ Contributions: (I) Conception and design: Q Tan, Y Xu; (II) Administrative support: L Feng; (III) Provision of study materials or patients: J Huang, \\ R Liu; (IV) Collection and assembly of data: Z Guo, J Ning; (V) Data analysis and interpretation: Y Xu; (VI) Manuscript writing: All authors; (VII) \\ Final approval of manuscript: All authors. \\ Correspondence to: Dr. Qiang Tan; Liang Feng. Shanghai Lung Tumor Clinical Medical Center, Shanghai Chest Hospital, Shanghai Jiao Tong \\ University School (SJTU), Shanghai 200030, China. Email: dr_tanqiang@sina.cn; liang-liang-1983@hotmail.com.
}

\begin{abstract}
Background: Uni-portal video-assisted thoracoscopic surgery (VATS) has become a popular type of thoracic surgery. However, improvements to the closure of the single drainage tube hole are still in need.

Methods: From February 2019 to May 2019, we included 50 patients who received uni-portal VATS for lung disease or mediastinal disease and simple continuous suture to strengthen the closure of intramuscle combined with removal-free stitches on the skin. Follow-up items included incision length, chest tube drainage amount, chest tube drainage time, incision effusion leakage, postoperative subcutaneous emphysema, postoperative pain score.

Results: A total of 50 patients were included in this study, including 23 males and 27 females, with an average age of $60.08 \pm 9.73$ years old. The mean drainage on the first day after operation was $236.56 \pm 141.50 \mathrm{~mL}$, while the mean pain score on the first day after operation was $4.16 \pm 1.70$. Among 50 patients, only two cases of subcutaneous emphysema occurred.
\end{abstract}

Conclusions: Applying innovative simple continuous suture to strengthen the closure of intra-muscle combined with removal-free stitches on the skin into the closure of uni-portal VATS is safe and feasible.

Keywords: Uni-portal; video-assisted thoracoscopic surgery (VATS); removal-free stitches

Submitted Oct 30, 2019. Accepted for publication Nov 05, 2019.

doi: $10.21037 / \mathrm{atm} .2019 .11 .62$

View this article at: http://dx.doi.org/10.21037/atm.2019.11.62

\section{Introduction}

Lung cancer is the leading cause of cancer-related death among men aged 40 years and older and women aged 60 years and older (1). There is a potential for earlier lung cancer diagnosis through screening with lowdose computed tomography, which has demonstrated a $20 \%$ reduction in lung cancer mortality (1). With the advancement of surgical technique and medical equipment, video-assisted thoracoscopic surgery (VATS) has gradually replaced the traditional open thoracic surgery, becoming the main stream of thoracic surgery (2). Uni-portal VATS for lung cancer surgery was first described in 2010 and has since been rapidly adopted around the world due to its advantages of less postoperative pain and cosmetic effect among other positives (3). The surgical approach is improving as time goes on; however, progress in the suture of the drainage tube hole has stagnated to the detriment of better outcomes. From February 2019 to May 2019, we included 50 patients who received uni-portal VATS for lung disease or mediastinal disease and had simple continuous suture to strengthen the closure of intra-muscle combined with removal-free stitches on the skin. We would like to share our experience with this innovative suture to help improve the general status of suture for VATS. 

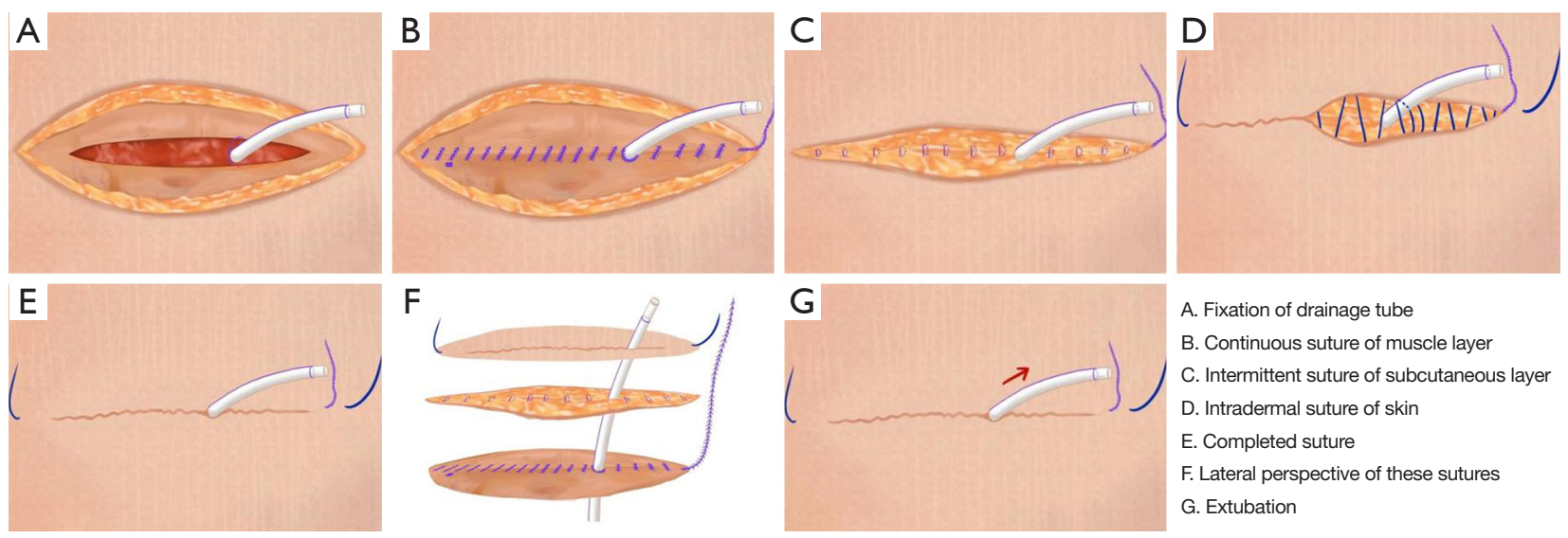

Figure 1 Stitching process. (A) Fixation of drainage tube; (B) continuous suture of muscle layer; (C) intermittent suture of subcutaneous layer; (D) intradermal suture of skin; (E) completed suture; (F) lateral perspective of these sutures; (G) extubation.

\section{Methods}

We conducted this innovative stitching method on 50 patients, who had received uni-portal thoracic surgery performed by one surgeon (Dr. Qiang Tan) for lobectomy, wedge resection, and mediastinal mastectomy in the Department of Pulmonary Oncology Surgery of the Shanghai Chest Hospital, Shanghai Jiao Tong University in China. Our team has extensive experience in conducting uni-portal thoracic surgery (4).

We only included patients who had already undergone uni-portal VATS surgery successfully and who were suitable to receive a placement of only a single $24 \mathrm{~F}$ common thoracic drainage tube without any intraoperative accidents like bleeding, anesthesia accidents, etc. Exclusion criteria included diabetes mellitus, scar tissue, secondary thoracic surgery, and intraoperative thoracotomy. The data of the patients included age, gender, surgical method, postoperative drainage time, postoperative hospitalization time, daily drainage volume, daily pain score of the incision (NRS score), infection of the incision, and wound healing status follow-up (3 weeks later) was recorded.

\section{Surgical technique}

\section{Fixation of drainage tube (Figure 1A)}

In the traditional suture method, many other incisions need to be made to place the drainage tube. At least 3 silk threads are required for the fixation of the chest tube. Each side of the drainage tube needs 1 thread to suture the wound and fix the tube. The last one is left without a knot, which is used for the closure of the drainage port when extubating.
In our innovative stitching method performed in this study, the thoracic drainage tube was placed directly in the incision as usual and close to the lower edge of the incision. However, a suture of 1-0 Vicryl was sewn into the muscle layer at the lowest edge of the incision, rather than sewn on the skin, and then a knot was tied about $5 \mathrm{~cm}$ away from the incision to fix the chest tube.

\section{Continuous suture of muscle layer (Figure 1B)}

After the thoracic drainage tube was fixed, the suture of the incision could begin. From the uppermost edge of the incision, conventional continuous suturing with Stratafix Symmetric (Ethicon LLC, Guaynabo, Puerto Rico), which is a mono-directional barbed wound-closure thread, was performed on the muscle layer. When suturing to the thoracic drainage tube, the tube should be bypassed, so that the thoracic drainage tube is located between the previous needle and the next needle. Finally, a $5-10 \mathrm{~cm}$ thread was left outside the skin.

\section{Intermittent suture of subcutaneous layer (Figure 1C)} Intermittent suturing with 1-0 Vicryl was performed on the subcutaneous layer from the uppermost to the bottom of the incision.

\section{Intradermal suture of skin (Figure 1D)}

Intradermal suturing with Stratafix Spiral (Ethicon LLC, Guaynabo, Puerto Rico), which is a 3-0 bidirectional barbed wound-closure thread, was performed on the skin. It was inserted about 1-2 $\mathrm{cm}$ away from the upper edge of the skin incision to the intradermal incision. Then, continuous 


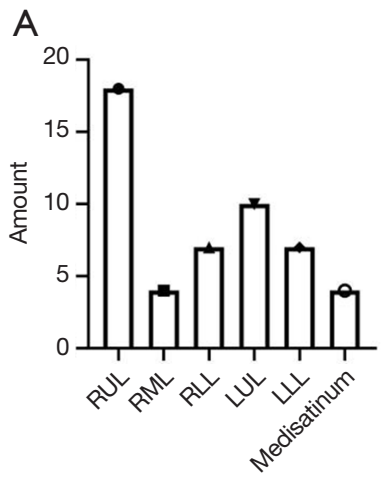

Surgical site

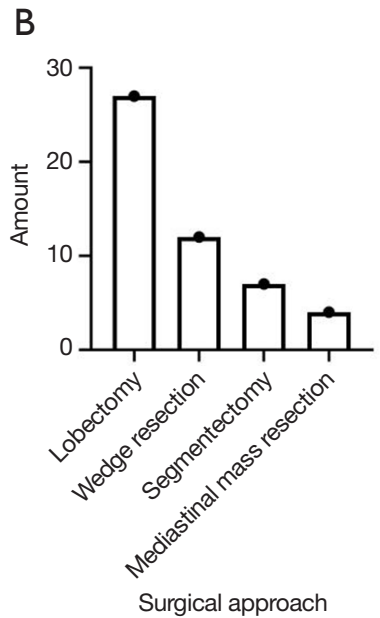

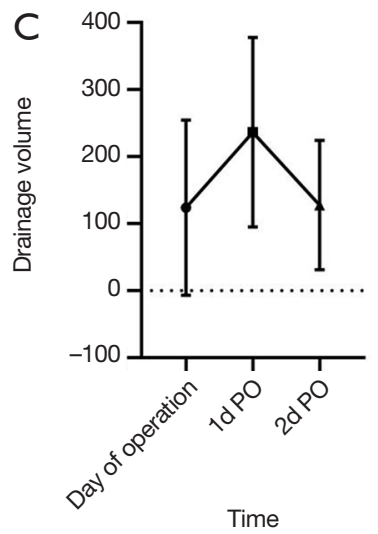

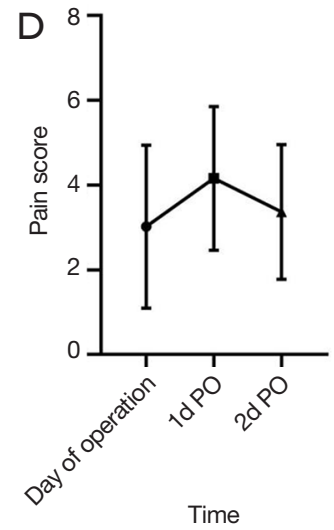

Figure 2 Summary of patient condition. (A) The amount of surgical site; (B) the amount of surgical approach; (C) postoperative drainage volume; (D) postoperative pain score.

intradermal suture was performed from the top edge of the incision to the bottom edge after bypassing the thoracic drainage tube. The last suture was inserted $1-2 \mathrm{~cm}$ away from the lower edge of skin incision with a $5-10 \mathrm{~cm}$ thread left outside the skin (Figure 1E). The lateral perspective of these sutures is shown in the Figure $1 F$.

\section{Extubation}

After the wound was disinfected, the fixed thread of the thoracic drainage tube was cut off, and the patient was instructed to inhale deeply and hold his breath. While removing drainage tube quickly and gently, tighten the mono-directional barbed wound-closure thread [Stratafix Symmetric (Ethicon LLC, Guaynabo, Puerto Rico)], which will strengthen the closure of muscle layer to avoid fluid or air leaks (Figure 1G). Then, the Stratafix Spiral (Ethicon LLC, Guaynabo, Puerto Rico) was tightened to close the drainage tube hole of skin. Finally, the 2 threads left above the skin were cut off.

\section{Follow-up}

An analgesic pump was routinely used on the day of surgery. Postoperative drainage was generally 2 to 3 days, and the flow rate was recorded. The NRS international pain scale was used to score the pain of each patient from the day after surgery to the day of extubating. Three weeks after the operation, the wound healing status was assessed, and the length of the wound was measured.

\section{Results}

A total of 50 patients were included in this study, including 23 males and 27 females, with an average age of $60.08 \pm 9.73$ years old. Among them, 27 patients underwent lobectomy, 7 patients underwent pulmonary segmentectomy, 12 patients underwent wedge resection, and 4 patients underwent mediastinal mass resection (Figure 2A,B).

The average length of postoperative hospitalization was $2.64 \pm 0.56$ days. The average drainage volume on the first day postoperative was $236.56 \pm 141.50 \mathrm{~mL}$. The average drainage volume on the second day postoperative was $127.90 \pm 96.58 \mathrm{~mL}$ (only 18 cases remained on drainage on the second day post operation). The average pain score on the day of operation was $3.02 \pm 1.92$ (analgesic pump was used for analgesic treatment on the day of operation). The mean pain score (assessed by the NRS pain scale) on the first day postoperative was $4.16 \pm 1.70$. The mean pain score on the second day postoperative was $3.36 \pm 1.59$ (1 patient was discharged from the hospital on the second postoperative day). The pain score on the third day was $3.36 \pm 1.59$ (19 patients were discharged on day 3 ). Subcutaneous emphysema occurred in 2 patients (Figure $2 C, D)$.

\section{Discussion}

The traditional suture for a drainage tube hole may result in intrathoracic fluid or air leaks into the subcutaneous tissue or air leaks from outside into thoracic when extubating, 
which also leaves an ugly scar on the skin (5). Conventional intradermal suture of skin also has to be removed, which not only affects patients' experience but also increase doctors' work burden, especially when complications like pneumothorax and wound infection emerge. Therefore, we created an innovative stitching method that applies simple continuous suture to strengthen the closure of the intramuscle combined with removal-free stitches on skin.

The traditional suture for drainage is performed with 3 threads. Each side of the drainage tube needs 1 thread to suture the wound and fix the tube. The last one is left without a knot, which is used for the closure of the drainage port when extubating. When extubating, two threads are cut off beside the tube, and the indwelling thread is quickly tied to close the hole in the skin. However, tying the indwelling thread only closes the hole in the skin, but does not suffice to close the drainage hole on the muscle. As a result, there is a good chance of intrathoracic fluid and air leak into the subcutaneous tissue and outside the skin, which may increase the risk of wound infection. It is also likely for the air outside the thoracic to run into the thorax resulting in pneumothorax.

For these reasons, we apply simple continuous suture to strengthen the closure of intra-muscle. At the time of extubating, we quickly tighten the tail of the monodirectional barbed wound-closure thread left outside the skin. Because this thread is designed to be tie-free, the drainage tube hole on intra-muscle can be closed without tying. Such quick tying will effectively strengthen the closure of the drainage tube hole on the muscle layer, solving the difficult problems of the traditional suture method. However, although we can apply continuous suture to strengthen the closure of the subcutaneous layer, the price of barbed wound-closure thread limits this method.

Intradermal suturing with 3-0 bidirectional barbed wound-closure thread is performed on the skin. After extubating and tightening the mono-directional barbed wound-closure thread, the bidirectional barbed woundclosure thread is tightened on the skin so that the drainage tube hole on skin can be closed. Applying this suture can bring cosmetic effect for patients and avoid scarring. This suture is also remove-free (6), which can also decrease doctors' work burden and improve patients' medical experience.

To evaluate the pain result from the suture, The NRS international pain scale was used to score the pain of each patient from the day after surgery to the day of extubating. The average pain score on the day of operation, postoperative day 1 , and postoperative day 2 were $3.02 \pm 1.92$, $3.36 \pm 1.59$ and $3.36 \pm 1.59$, respectively. This shows that the pain was tolerable for patients, and our new approach does not entail additional pain.

This method relies on the characteristic of tie-free barbed suture thread so that we can strengthen the closure of intra-muscle and skin without tying (7). Barbed suture and Vicryl suture have been proven to be safe and are widely used across the world $(8,9)$. Though this suture method shows many advantages, its data lacks comparative design with traditional suture. Adequate follow-up period time is also needed to be proved the effect of this suture.

Fifty patients underwent this innovative suture to close the incision. Only 2 cases of subcutaneous emphysema occurred. After 3 weeks of follow-up, most patients were satisfied with the healing of their incision. Uni-portal VATS has significant advantages including less postoperative pain (10), less skin numbness, less paresthesia, quicker recovery, better cosmetology and stronger psychological comfort especially for young women $(11,12)$. Applying simple continuous suture to strengthen the closure of intra-muscle combined with removal-free stitches on skin with uni-portal VATS proves to a safe and feasible suture method.

\section{Acknowledgments}

Funding: This project was supported by the National Natural Science Foundation of China (81871497).

\section{Footnote}

Conflicts of Interest: The authors have no conflicts of interest to declare.

Ethical Statement: The authors are accountable for all aspects of the work in ensuring that questions related to the accuracy or integrity of any part of the work are appropriately investigated and resolved. The study was approved by Ethics Committee of Shanghai Chest Hospital [No. KS $(Y) 1835]$ and written informed consent was obtained from all patients.

\section{References}

1. Siegel RL, Miller KD, Jemal A. Cancer statistics, 2019. CA Cancer J Clin 2019;69:7-34.

2. Luh SP, Liu HP. Video-assisted thoracic surgery--the 
past, present status and the future. J Zhejiang Univ Sci B 2006;7:118-28.

3. Sihoe ADL. Uniportal Lung Cancer Surgery: State of the Evidence. Ann Thorac Surg 2019;107:962-72.

4. Liu R, Tan Q, Huang J, et al. Uniportal video assisted thoracic surgery with $2 \mathrm{~cm}$ skin incision for right middle lobectomy with systematic lymphadenectomy. J Thorac Dis 2017;9:4592-6.

5. Son BS, Park JM, Seok JP, et al. Modified incision and closure techniques for single-incision thoracoscopic lobectomy. Ann Thorac Surg 2015;99:349-51.

6. Paul MD. Barbed sutures in aesthetic plastic surgery: evolution of thought and process. Aesthet Surg J 2013;33:17S-31S.

7. Mulholland RS, Paul MD. Lifting and wound closure with barbed sutures. Clin Plast Surg 2011;38:521-35, viii.

8. Moya AP. Barbed sutures in body surgery. Aesthet Surg J

Cite this article as: $\mathrm{Xu} \mathrm{Y,} \mathrm{Guo} \mathrm{Z,} \mathrm{Huang} \mathrm{J,} \mathrm{Liu} \mathrm{R,} \mathrm{Ning} \mathrm{J,}$ Feng L, Tan Q. Simple continuous suture to strengthen the closure of intra-muscle used in the removal of uni-portal videoassisted thoracoscopic surgery thoracic drainage tube. Ann Transl Med 2019;7(23):764. doi: 10.21037/atm.2019.11.62
2013;33:57S-71S.

9. Mahesh L, Kumar VR, Jain A, et al. Bacterial Adherence Around Sutures of Different Material at Grafted Site: A Microbiological Analysis. Materials (Basel) 2019. doi: 10.3390/ma12182848.

10. Zhu Y, Liang $M, W u ~ W$, et al. Preliminary results of single-port versus triple-port complete thoracoscopic lobectomy for non-small cell lung cancer. Ann Transl Med 2015;3:92.

11. Yang HC, Noh D. Single incision thoracoscopic lobectomy through a $2.5 \mathrm{~cm}$ skin incision. J Thorac Dis 2015;7:E122-5.

12. Hirai K, Takeuchi S, Usuda J. Single-incision thoracoscopic surgery and conventional video-assisted thoracoscopic surgery: a retrospective comparative study of perioperative clinical outcomesdagger. Eur J Cardiothorac Surg 2016;49 Suppl 1:i37-41. 